\title{
Cancer incidence according to the National Health Information Database in Korean patients with end-stage renal disease receiving hemodialysis
}

\author{
Jisun Myung ${ }^{1}$, Jung Hye Choi ${ }^{2}$, Joo Hark $\mathrm{Yi}^{2}$, and Inah Kim
}

${ }^{1}$ Biostatistical Consulting and Research Laboratory, Medical Research Coordinating Center, Hanyang University, Seoul; ${ }^{2}$ Department of Internal Medicine, Hanyang University Guri Hospital, Guri; ${ }^{3}$ Department of Occupational and Environmental Medicine, Hanyang University College of Medicine, Seoul, Korea

Received: November 9, 2018

Revised : May 8, 2019

Accepted: May 21, 2019

Correspondence to

Jung Hye Choi, M.D.

Department of Internal

Medicine, Hanyang University

Guri Hospital, 153 Gyeongchun-

ro, Guri 11923, Korea

Tel: +82-31-560-2162

Fax: +82-31-553-7369

E-mail:jhcmd@hanyang.ac.kr
Background/Aims: The aim of this study was to investigate incidence, survival, and risk factors of cancer in end-stage renal disease (ESRD) patients with hemodialysis using information from the National Health Information Database (NHID). Methods: Using the NHID, we identified ESRD patients who started maintenance hemodialysis between 2003 and 2005 in Korea. Patients were followed from initiation of hemodialysis to renal transplantation, death, or December 31, 2016, whichever came first. We calculated the incidence, survival, and risk factors of cancer.

Results: Of the total 14,382 ESRD patients, 1,124 (7.82\%; men:women, 728:396) were diagnosed with cancer during follow-up. The mean duration from the start of hemodialysis to new cancer identification was $64.40 \pm 41.81$ months. Significant risk factors for the development of new cancer were old age, male sex, and liver disease. Conversely, patients with diabetes showed low risk for new cancer. The colorectum (17.31\%) was the most common primary site of cancer in men, followed by the liver (15.8\%), stomach (14.29\%), lung (13.6\%), and kidney (10.3\%). In women, the colorectum (14.65\%) was also the most common primary site of cancer, followed by the breast (12.88\%), thyroid (12.63\%), stomach (10.86\%), and lung (8.08\%). According to the primary site of cancer, breast cancer showed the longest median survival duration (130.93 months), followed by thyroid, kidney, colorectum, bladder, stomach, liver, and lung cancer. On multivariate analyses, overall survival was affected by age and diabetes.

Conclusions: The cancer incidence of chronic hemodialysis patients was relatively high. Thus, careful monitoring and a specific cancer screening program are needed for chronic hemodialysis patients.

Keywords: Renal dialysis; Kidney failure, chronic; Neoplasms

\section{INTRODUCTION}

The incidence of end-stage renal disease (ESRD) is rapidly increased worldwide [1]. Dialysis has been considered a standard therapy for patients with ESRD unless kidney transplantation is possible. Therefore, the number of patients on maintenance dialysis continues to grow.
Although cardiovascular disease is the most common cause of mortality in ESRD patients, cancer may be a main cause of death in this population due to improved dialysis methods and increased life spans [2]. Outcomes of cancer treatment are substantially improved due to the development of new drugs and improvements in supportive care; however, treatment for ESRD cancer 
patients has not been established because most clinical trials exclude ESRD patients.

Although cancer in ESRD patients is an important emerging medical issue, there are only two reports on cancer in dialysis patients in Korea. A retrospective, single-center study showed that 106 patients (2.3\%) out of 4,562 ESRD patients on chronic maintenance hemodialysis or peritoneal dialysis from 1996 to 2005 were diagnosed with cancer [3]. The mean time from initiation of dialysis to cancer diagnosis was 75.2 \pm 63.9 months. Another observational cohort study reported that $116(2.2 \%)$ of 5,235 ESRD patients were diagnosed with cancer, and the standardized incidence ratio (SIR) was highest for urinary tract cancer [4].

The aim of this study was to investigate incidence, survival, and risk factors of cancer in patients treated by hemodialysis using the National Health Information Database (NHID). Based on our results, we plan to construct cancer screening guidelines for patients with hemodialysis in Korea.

\section{METHODS}

\section{Sources of data}

All data were collected from the National Health Insurance Service (NHIS) using their customized database service. In Korea, nearly all citizens have joined the National Health Insurance program as a mandatory social insurance since 1989. The NHID is a public database formed by the NHIS and contains sociodemographic characteristics, dates of birth and death, health care utilization, and health screening for the whole population of Korea [5]. Therefore, we used the NHID to obtain the most accurate epidemiological data of the entire Korean population.

This study was approved by the Institutional Review Board of Hanyang University Guri Hospital (2017-10o03). Because personal information in this national dataset was de-identified for research use, the committee waived the requirement of written informed consent.

\section{Selection of patients}

We initially selected end stage renal disease patients who had started maintenance hemodialysis between January 1, 2003 and December 31, 2005. Patients who started hemodialysis were identified as those having a new claim of hemodialysis-related code, including Voo1, O7020, O7021, or O9991. Among them, we defined ESRD patients on maintenance hemodialysis as receiving hemodialysis for more than 90 days. The key exclusion criteria were a history of any cancer and/or transplantation before dialysis, age of 18 years or younger, claims of hemodialysis only as an inpatient condition, and patients who survived for less than 90 days from initiation of dialysis. Patients were followed from the time of initiation of hemodialysis to renal transplantation, death, or December 31, 2016, whichever came first.

\section{Main outcome measurements}

In Korea, patients with newly diagnosed malignant disease were registered with a special certification for cancer, code V193, from September 2005 to reduce the cost. Cancer was defined as a new claim for major diagnosis of C code (ICD 10: Coo-C96) with the inpatient condition until August 2005; thereafter (from September 2005), it was defined as a new claim for major diagnosis of C code and V193. The cancer occurrence date was defined as the date of a new claim. Patients who had two or more codes for cancer were considered diagnosed with the first claimed code. The definition of newly diagnosed cancer was a diagnosed malignant neoplasm at least 90 days after initiation of hemodialysis.

\section{Covariate assessment}

To analyze risk factors associated with cancer occurrence, we obtained data on age at initiation of hemodialysis, sex, insurance type, and comorbidities. Insurance type was used as an indirect indicator of income. The ICD-1o codes used to define potential confounding comorbidities were as follows: diabetes mellitus (E101, E102, E103, E104, E105, E109, E111, E112, E113, E114, E115, E119, E131, E132, E133, E134, E135, E139, E141, E142, E143, E144, E145, E149), hypertension (I10), liver disease (B18, $\mathrm{K}_{702}, \mathrm{~K}_{703}, \mathrm{~K}_{717}, \mathrm{~K}_{73}, \mathrm{~K}_{740}$, K742, K743, K744, K745, K746, $\mathrm{K}_{721}, \mathrm{~K}_{729}, \mathrm{~K}_{766}, \mathrm{~K}_{767}$ ), pulmonary disease (J40-J47, J60-J67), and cardiovascular disease, including angina, acute myocardial infarction, congestive heart failure, peripheral vascular disease, or cerebral vascular accident (I20, I21, I22, I252, I50, I71, I790, I739, Ro2, Z958, Z959, I6o, I61, I62, I63, I64, I65, I66, I670, I671, I672, I674, I675, I676, I677 I678, I679, I681, I682, I688, I69, G450, G451, 
$\mathrm{G}_{452}, \mathrm{G}_{454}, \mathrm{G}_{45} 8, \mathrm{G}_{459}, \mathrm{G}_{46}$ ). We referred to new ICD-10 version of the Charlson comorbidity index for defining these comorbidities [6]. We added chronic viral hepatitis $(\mathrm{B} 18)$ to liver disease because Korea is endemic area of hepatitis B. These comorbidities were identified in more than two hospital outpatient visits or more than one hospital inpatient visit using diagnostic codes within 1 year before the start of dialysis.

\section{Statistical analysis}

Statistical analyses were performed using SAS version 9.4 (SAS Institute, Cary, NC, USA). Data are presented as the mean \pm standard deviation (SD). The Pearson chisquare test was performed to compare covariates between the groups with and without a new cancer diagnosis. Incidence rate was calculated. Hazard ratio (HR) and $95 \%$ confidence interval (CI) were calculated.

Overall survival was measured from the date of cancer diagnosis to the date of death from any cause or the date of last follow-up. Survival curves were calculated using the Kaplan-Meier method and compared with other prognostic variables using the log-rank test. The Cox proportional hazards model was performed to identify prognostic factors for survival. A $p<0.05$ was considered statistically significant.

\section{RESULTS}

\section{Characteristics of the cohort}

We initially selected a total of 49,770 ESRD patients with hemodialysis-related codes, including Voo1, O7020, O7021, or O9991, between January 1, 2003 and December 31, 2005. Among them, we excluded 35,388 patients: 24,333 with less than 90 days duration between the initial and last claims of hemodialysis, 10,341 who started hemodialysis before 2003, 346 who had a history of cancer, 193 who had undergone transplantation before dialysis, 94 with missing data on date of birth or sex, and 81 who were 18 years or younger. Finally, we identified 14,382 ESRD patients who started maintenance hemodialysis between 2003 and 2005. The mean follow-up duration was $77.51 \pm 53.99$ months.

Patient characteristics are summarized in Table 1. The mean age at the time of initial hemodialysis was $58.22 \pm$ 12.15 years. Hypertension (70.0\%) was the most common comorbidity, followed by diabetes mellitus (42.9\%), cardiovascular disease (33.7\%), pulmonary disease (15.4\%), and liver disease (7.1\%). A total of 2,186 patients (15.2\%) underwent renal transplantation at a mean of $55.56 \pm 43.13$ months after initiation of hemodialysis. Among the 14,382 ESRD patients, 8,883 (61.76\%) died during follow-up.

\section{Occurrence and risk factors of cancer}

Of the total 14,382 ESRD patients who started maintenance hemodialysis between 2003 and 2005, 1,124 (7.82\%) were diagnosed with cancer during the study period. Among them, 97 patients (8.63\%) had two or more codes for cancer, and they were considered diagnosed with the first claimed code. Cancer patients included 728 men and 396 women, and the mean age at cancer diagnosis was $63.43 \pm 11.56$ years. The mean duration from the start of hemodialysis to new cancer identification was $64.40 \pm$ 41.81 months, and the median was 56.92 months (range, 3.07 to 168.23). The significant risk factors for the development of new cancer were old age, male sex, and liver disease. On the contrary, patients with diabetes showed low risk for new cancer (Table 2).

Cancer incidence according to the duration of hemodialysis is shown in Fig. 1. Cumulative incidence of any cancer from the start of hemodialysis in ESRD patients, stratified by sex is described in Fig. 2 .

\section{Primary site of cancer}

The primary site of cancer classified by sex is reported in Table 3. In the 728 male patients, the colorectum (17.31\%) was the most common primary site of cancer, followed by the liver (15.8\%), stomach (14.29\%), lung (13.6\%), kidney (10.3\%), bladder (5.35\%), and prostate (4.26\%). The mean time from start of hemodialysis to cancer development was $60 \pm 41.01$ months. Hematologic malignancy had the shortest mean duration of cancer development ( $45.29 \pm 46.53$ months). In the 396 female cancer patients, the colorectum (14.65\%) was also the most common primary site of cancer, followed by the breast (12.88\%), thyroid (12.63\%), stomach (10.86\%), lung (8.08\%), uterus (7.58\%), and kidney (6.57\%). The mean duration from start of hemodialysis to cancer development was $70.83 \pm 42.54$ months in women.

\section{Survival analyses of hemodialysis patients with cancer}

The median survival duration of 14,382 ESRD patients 
Table 1. Comparison of baseline characteristics between end-stage renal disease patients with or without cancer $(n=14,382)$

\begin{tabular}{|c|c|c|}
\hline Characteristic & $\begin{array}{l}\text { Without new cancer } \\
(\mathrm{n}=13,258,92.18 \%)\end{array}$ & $\begin{array}{l}\text { With new cancer } \\
(\mathrm{n}=1,124,7.82 \%)\end{array}$ \\
\hline \multicolumn{3}{|l|}{ Age at first dialysis, yr } \\
\hline $19-49$ & $3,871(93.66)$ & $262(6.34)$ \\
\hline $50-59$ & $3,180(91.27)$ & $304(8.73)$ \\
\hline $60-69$ & $3,913(91.53)$ & $362(8.47)$ \\
\hline$\geq 70$ & $2,294(92.13)$ & $196(7.87)$ \\
\hline Mean age at first dialysis $(n=14,382), y r$ & \multicolumn{2}{|c|}{$58.22 \pm 12.15$} \\
\hline Median age at first dialysis $(\mathrm{n}=14,382), \mathrm{yr}$ & \multicolumn{2}{|c|}{59} \\
\hline Mean age at cancer diagnosed $(n=1,124), y r$ & \multicolumn{2}{|c|}{$63.43 \pm 11.56$} \\
\hline Median age at cancer diagnosed $(n=1,124), y r$ & \multicolumn{2}{|c|}{65} \\
\hline Mean time from dialysis to cancer $(n=1,124)$, mon & \multicolumn{2}{|c|}{$64.40 \pm 41.81$} \\
\hline Median time from dialysis to cancer $(n=1,124)$, mon & \multicolumn{2}{|c|}{56.92} \\
\hline \multicolumn{3}{|l|}{ Sex } \\
\hline Female & $5,857(93.67)$ & $396(6.33)$ \\
\hline Male & $7,401(91.04)$ & $728(8.96)$ \\
\hline \multicolumn{3}{|l|}{ Insurance } \\
\hline Yes & $12,585(92.14)$ & $1,073(7.86)$ \\
\hline No (medical aid beneficiaries) & $673(92.96)$ & $51(7.04)$ \\
\hline \multicolumn{3}{|l|}{ Comorbidity } \\
\hline \multicolumn{3}{|l|}{ Diabetes mellitus } \\
\hline No & 7,488 (91.19) & $723(8.81)$ \\
\hline Yes & $5,770(93.50)$ & $401(6.50)$ \\
\hline \multicolumn{3}{|l|}{ Liver disease } \\
\hline No & $12,352(92.43)$ & $1,012(7 \cdot 57)$ \\
\hline Yes & $906(89.00)$ & $112(11.00)$ \\
\hline \multicolumn{3}{|l|}{ Hypertension } \\
\hline No & $3,966(92.00)$ & $345(8.00)$ \\
\hline Yes & $9,292(92.26)$ & $779(7.74)$ \\
\hline \multicolumn{3}{|l|}{ Cardiovascular disease } \\
\hline No & $8,775(92.03)$ & $760(7.97)$ \\
\hline Yes & $4,483(92.49)$ & $364(7 \cdot 51)$ \\
\hline \multicolumn{3}{|l|}{ Lung disease } \\
\hline No & $11,213(92.12)$ & $959(7.88)$ \\
\hline Yes & $2,045(92.53)$ & $165(7.47)$ \\
\hline
\end{tabular}

Values are presented as number $(\%)$ or mean \pm SD.

from the initiation of dialysis was 86.37 months (range, 0.5 to 170.23). The median survival duration of ESRD patients with cancer was significantly shorter than that of ESRD patients without cancer (72.63 months vs. 87.5 months, $p<0.001$ ) (Fig. 3).

Of the 1,124 ESRD patients with cancer, the median survival duration from date of cancer diagnosis was
26.63 months. According to the primary site of cancer, breast cancer showed the longest median survival duration (130.93 months), followed by thyroid (105.87), kidney (78.73), colorectum (35.33), bladder (30.9), stomach (23.33), liver (9.4), and lung (6.7).

On univariate analysis using the log-rank test, significant risk factors for overall survival in ESRD patients 
Table 2. Risk factors for development of new cancer in end-stage renal disease patients with chronic maintenance hemodialysis

\begin{tabular}{lc}
\hline Variable & Multivariate analysis \\
\cline { 2 - 2 } Age at first dialysis, yr & Adjusted HR $(95 \%$ CI $)$ \\
\hline $19-49$ & $1($ reference) \\
50-59 & $1.599(1.353-1.891)^{\mathrm{a}}$ \\
60-69 & $1.93(1.638-2.275)^{\mathrm{a}}$ \\
$\quad \geq 70$ & $2.697(2.223-3.272)^{\mathrm{a}}$ \\
Sex (male vs. female) & $1.529(1.352-1.729)^{\mathrm{a}}$ \\
National Health Insurance (medical aid beneficiaries vs. subscribers) & $0.902(0.67-1.214)$ \\
Diabetes mellitus (yes vs. no) & $0.853(0.746-0.975)^{\mathrm{a}}$ \\
Liver disease (yes vs. no) & $1.57(1.29-1.912)^{\mathrm{a}}$ \\
Hypertension (yes vs. no) & $0.975(0.845-1.126)$ \\
Cardiovascular disease (yes vs. no) & $1.126(0.983-1.29)$ \\
\hline Lung disease (yes vs. no) & $1.023(0.864-1.211)$ \\
\hline
\end{tabular}

Using the Cox proportional hazards model.

$\mathrm{HR}$, hazard ratio; CI, confidence interval.

${ }^{a}$ Significantly different.

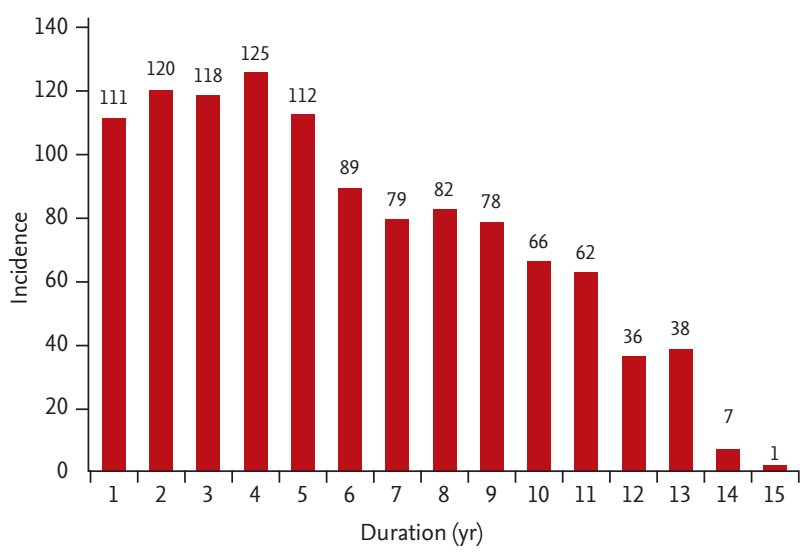

Figure 1. Cancer incidence according to duration of hemodialysis.

with cancer were age $\geq 50$, male, diabetes mellitus, cardiovascular disease, and lung disease (Fig. 4). Although women had a longer survival duration than men, there was no significant survival difference between women and men for major cancers, including stomach, colon, liver, kidney, bladder, thyroid, and lung cancer. On multivariate analyses, overall survival was affected by age and diabetes (Table 4).

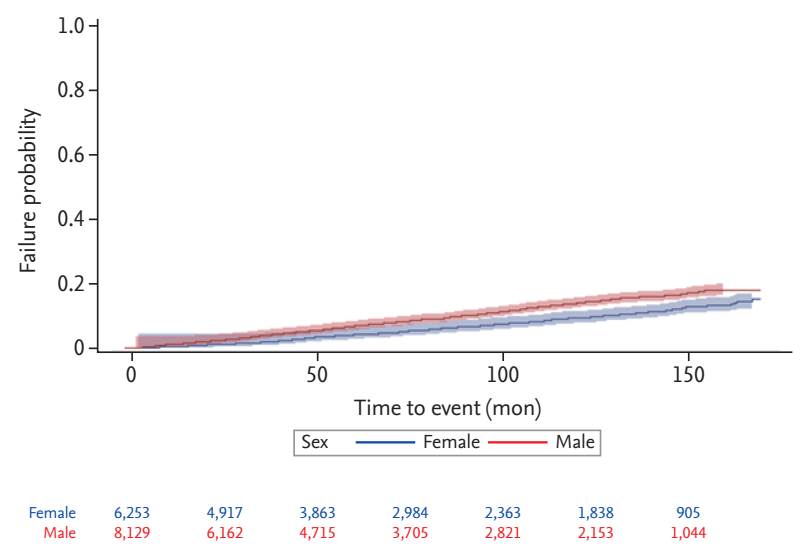

Figure 2. The cumulative incidence of any cancer from the start of hemodialysis in end-stage renal disease patients, stratified by sex.

\section{DISCUSSION}

This is the first study to investigate incidence, survival, and risk factors of cancer in ESRD patients with chronic maintenance hemodialysis using a nationwide population-based database, the NHID, which includes the entire Korean population. We found that 1,124 (7.82\%) of 14,382 ESRD patients who started maintenance hemodialysis between 2003 and 2005 were diagnosed with 
Table 3. Site-specific cancer incidence, mean time from dialysis to cancer, and incidence rate of cancers

\begin{tabular}{|c|c|c|c|c|c|c|}
\hline \multirow[b]{2}{*}{ Variable } & \multicolumn{3}{|c|}{ Men } & \multicolumn{3}{|c|}{ Women } \\
\hline & No. (\%) & $\begin{array}{c}\text { Mean time from } \\
\text { dialysis to cancer, mon }\end{array}$ & $\begin{array}{l}\text { Incidence } \\
\text { rate }^{\mathrm{a}}\end{array}$ & No. (\%) & $\begin{array}{c}\text { Mean time from } \\
\text { dialysis to cancer, mon }\end{array}$ & $\begin{array}{l}\text { Incidence } \\
\text { rate }^{\mathrm{a}}\end{array}$ \\
\hline Total cancers & $728(100)$ & $60.9 \pm 41.01$ & $1,420.48$ & $396(100)$ & $70.83 \pm 42.54$ & 950.89 \\
\hline Head and neck & $18(2.47)$ & $51.02 \pm 40.77$ & 35.12 & $4(1.01)$ & $54.08 \pm 17.07$ & 9.605 \\
\hline Stomach & $104(14.29)$ & $56.79 \pm 40.09$ & 202.93 & $43(10.86)$ & $65.67 \pm 44.52$ & 103.253 \\
\hline Colon & $126(17.31)$ & $58.63 \pm 37.61$ & 245.85 & $58(14.65)$ & $64.47 \pm 39.98$ & 139.272 \\
\hline Liver & $115(15.80)$ & $55.15 \pm 40.04$ & $224 \cdot 39$ & $20(5.05)$ & $74.48 \pm 37.65$ & 48.025 \\
\hline Pancreatobiliary & $21(2.88)$ & $65.69 \pm 39.62$ & 40.98 & $17(4.29)$ & $64.41 \pm 74.48$ & 40.821 \\
\hline Lung & $99(13.60)$ & $56.42 \pm 40.74$ & 193.17 & $32(8.08)$ & $80.64 \pm 40.87$ & 76.84 \\
\hline Skin & $21(2.88)$ & $79.99 \pm 38.36$ & 40.98 & $13(3.28)$ & $66.89 \pm 37.75$ & 31.216 \\
\hline Breast & $1(0.14)$ & $121.57 \pm 0.00$ & 1.95 & $51(12.88)$ & $79.82 \pm 46.62$ & 122.463 \\
\hline Uterus & 0 & - & - & $30(7.58)$ & $54 \cdot 51 \pm 34 \cdot 73$ & 72.037 \\
\hline Prostate & $31(4.26)$ & $52.02 \pm 39.41$ & 60.49 & o & - & - \\
\hline Kidney & $75(10.30)$ & $78.45 \pm 39.81$ & 146.34 & $26(6.57)$ & $97.07 \pm 43.96$ & 62.432 \\
\hline Bladder & $39(5 \cdot 36)$ & $65.2 \pm 43.22$ & 76.1 & $18(4 \cdot 55)$ & $55.25 \pm 36.40$ & 43.222 \\
\hline Thyroid & $26(3.57)$ & $83.89 \pm 37.54$ & 50.73 & $50(12.63)$ & $73.99 \pm 38.93$ & 120.062 \\
\hline Hematology & $22(3.02)$ & $45.29 \pm 46.53$ & 42.93 & $14(3 \cdot 54)$ & $56.44 \pm 38.73$ & 33.617 \\
\hline Other cancers & $30(4.12)$ & $59.99 \pm 46.66$ & 58.53 & $20(5.05)$ & $75.93 \pm 48.62$ & 48.02 \\
\hline
\end{tabular}

Values are presented as number (\%) or mean \pm SD.

a 100,000 person-years.

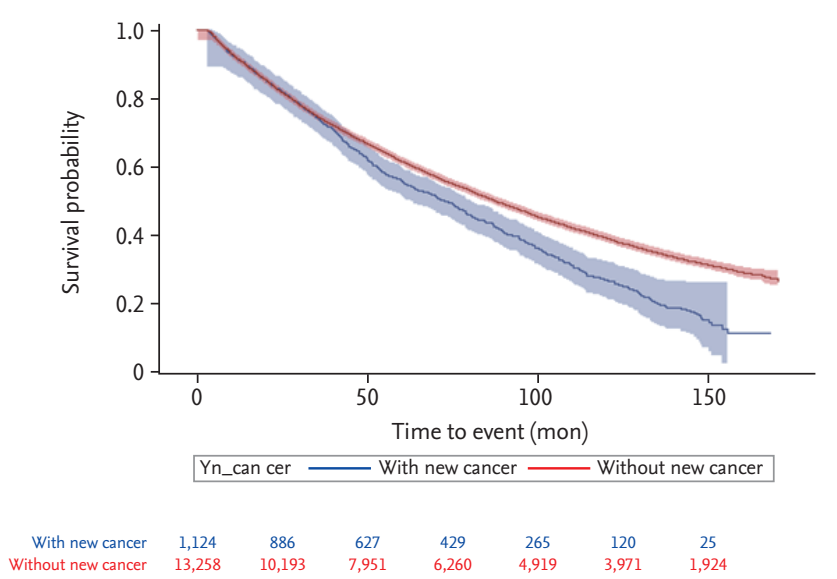

Figure 3. The overall survival of end-stage renal disease patients with cancer or without cancer.

cancer with mean follow-up duration of $77.51 \pm 53.99$ months. The mean duration from the start of hemodialysis to new cancer identification was $64.40 \pm 41.81$ months. The significant risk factors for development of new cancer were old age, male sex, and liver disease.
The colorectum was the most common primary site of cancer, and breast cancer showed the longest median survival duration.

There have been important studies on cancer incidence in dialysis patients. A large international collaboration study in the United States, Europe, Austria, and New Zealand reported that 25,044 (3\%) of 831,804 patients on dialysis for ESRD developed cancer during average follow-up of 2.5 years [7]. They concluded that the overall risk of cancer was increased in ESRD patients with a SIR of 1.18. They observed a high risk of cancer in a young age group, and kidney, bladder, and thyroid cancer showed a high SIR.

In a report on cancer incidence among United States Medicare ESRD patients on hemodialysis between 1996 and 2009, the 5-year crude cumulative incidence of cancer was $9.48 \%$ and was higher in subgroups of older age, male, and non-diabetes primary ESRD cause [8]. Although the cancer incidence identified in their study was higher than our results, the risk factors of cancer were similar to ours. 


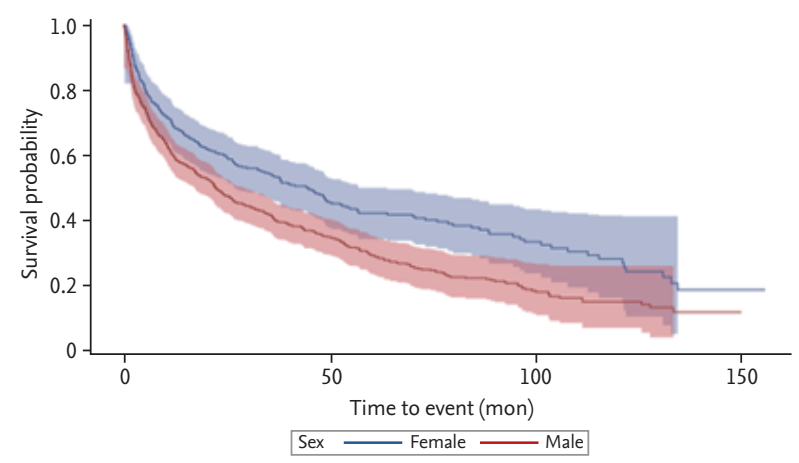

A
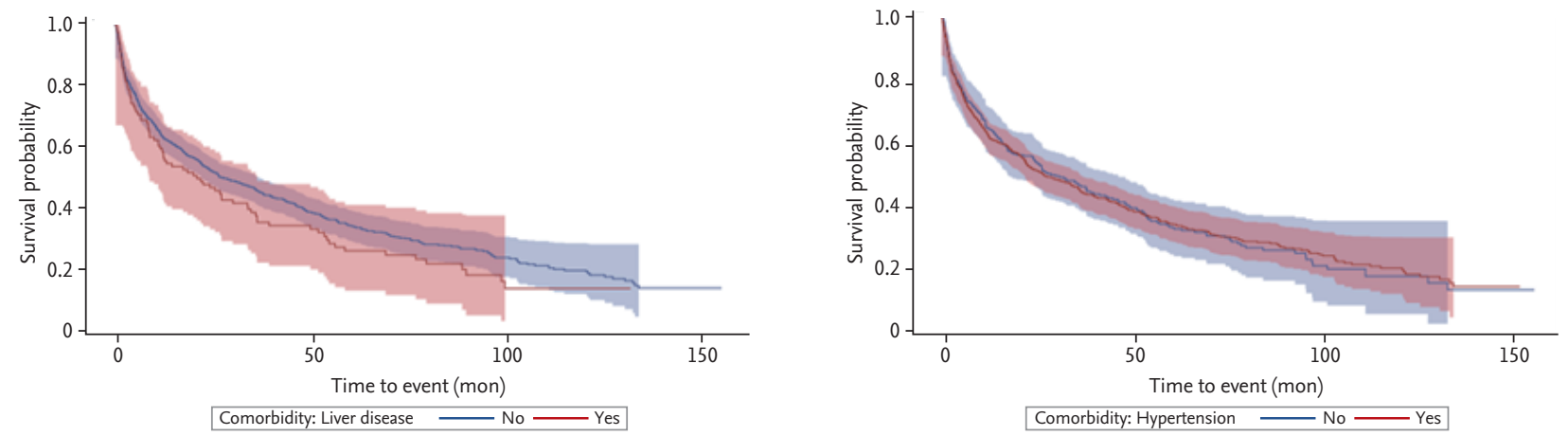

C

$\begin{array}{cccccccc}\text { No } & 1012 & 448 & 256 & 151 & 74 & 32 & 2 \\ \text { Yes } & 112 & 47 & 30 & 18 & 7 & 1 & 0\end{array}$
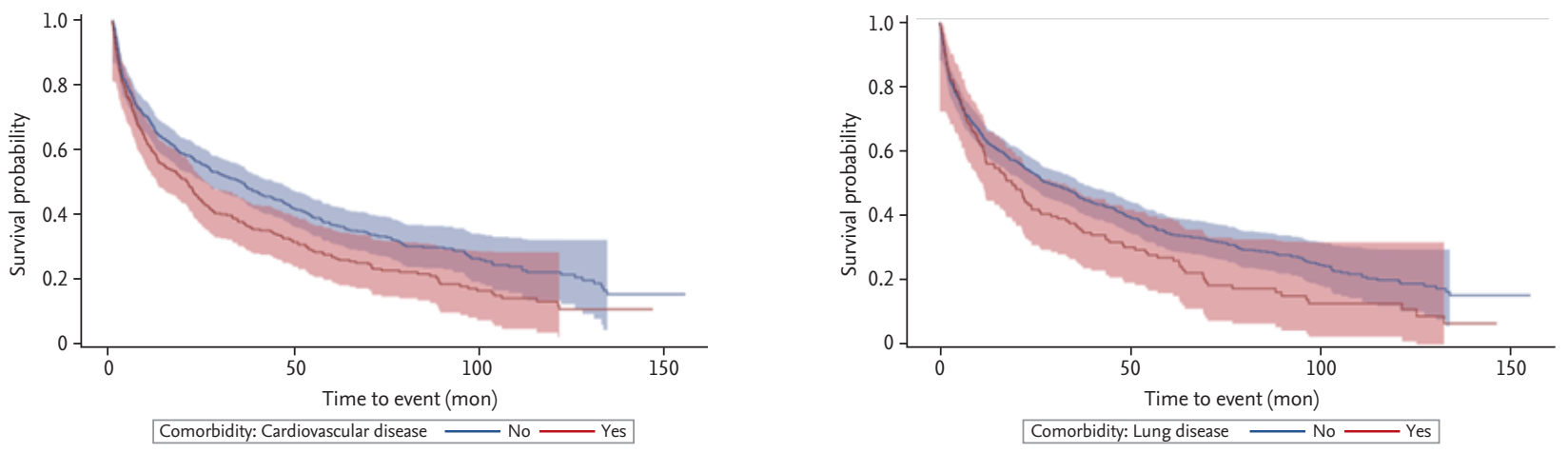

E

$\begin{array}{cccccccc}\text { No } & 760 & 356 & 204 & 120 & 58 & 26 & 2 \\ \text { Yes } & 364 & 139 & 82 & 49 & 23 & 7 & 0\end{array}$

$\mathbf{F}$

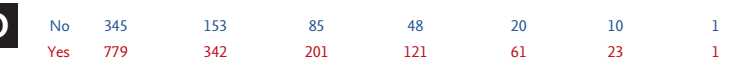

Figure 4. Kaplan-Meier plots of overall survival after new cancer diagnosis in 1,124 end-stage renal disease patients according to risk factors of (A) sex, (B) diabetes mellitus, (C) liver disease, (D) hypertension, (E) cardiovascular disease, and (F) lung disease. 
Table 4. Prognostic factors for overall survival in hemodialysis patients with cancer

\begin{tabular}{|c|c|c|}
\hline \multirow{2}{*}{ Variable } & Univariate analysis & Multivariate analysis \\
\hline & $\operatorname{HR}(95 \% \mathrm{CI})$ & $\operatorname{HR}(95 \% \mathrm{CI})$ \\
\hline \multicolumn{3}{|l|}{ Age at cancer diagnosis, yr } \\
\hline $19-49$ & 1 (reference) & 1 (reference) \\
\hline $50-59$ & $2.248(1.597-3.164)^{\mathrm{a}}$ & $1.909(1.348-2.702)^{\mathrm{b}}$ \\
\hline $60-69$ & $3.448(2.498-4.757)^{\mathrm{a}}$ & $3.056(2.184-4.276)^{\mathrm{a}}$ \\
\hline$\geq 70$ & $5.262(3.811-7.264)^{a}$ & $4.657(3.31-6.552)^{\mathrm{a}}$ \\
\hline Sex (male vs. female) & $1.442(1.233-1.687)^{\mathrm{a}}$ & $1.058(0.888-1.26)$ \\
\hline $\begin{array}{l}\text { National Health Insurance } \\
\text { (medical aid beneficiaries vs. subscribers) }\end{array}$ & $1.034(0.736-1.452)$ & $1.445(0.999-2.09)$ \\
\hline Diabetes mellitus (yes vs. no) & $1.482(1.281-1.715)^{\mathrm{a}}$ & $1.445(1.23-1.698)^{\mathrm{a}}$ \\
\hline Liver disease (yes vs. no) & $1.202(0.958-1.509)$ & $1.152(0.898-1.478)$ \\
\hline Hypertension (yes vs. no) & $1.003(0.858-1.172)$ & $0.731(0.609-0.878)^{b}$ \\
\hline Cardiovascular disease (yes vs. no) & $1.323(1.139-1.537)^{\mathrm{a}}$ & $1.14(0.969-1.341)$ \\
\hline Lung disease (yes vs. no) & $1.289\left(1.065^{-1.559)^{b}}\right.$ & $0.983(0.808-1.196)$ \\
\hline
\end{tabular}

Using the Cox proportional hazards model.

$\mathrm{HR}$, hazard ratio; CI, confidence interval.

${ }^{\mathrm{a}} \mathrm{p}<0.001$.

${ }^{\mathrm{b}} \mathrm{p}<0.05$.

In Taiwan, the Taiwan National Health Insurance program has provided universal health insurance for nearly all of Taiwan's residents since 1995 [9]. Recently, several studies were published on the epidemiology and risk of cancer in dialysis patients using data from Taiwan's National Health Insurance Research Database [911]. One important study identified ESRD patients starting maintenance dialysis between 1999 and 2004 and then followed the patients from initiation of dialysis to death, end of dialysis, or the end of 2008 . Of the 40,833 patients, 2,352 (5.8\%) developed cancer. The mean duration from start of hemodialysis to new cancer identification was $41.29 \pm 26.58$ months. The independent risk factors for the development of new cancer were older age, male, and chronic liver disease. Patients with diabetes, hypertension, or cardiovascular disease showed lower risk for new cancer. Liver cancer (20.63\%) and bladder cancer $(25.57 \%)$ were the most common cancers in men and women, respectively [9].

The study design and results of this report were like ours except that the Taiwan study included patients with peritoneal dialysis. Both studies selected ESRD pa- tients with initiation of maintenance dialysis for a specific period and then identified patients observed until a pre-selected date using a National Health Insurance Database. The risk factors for the development of cancer were similar in the two studies. While diabetes mellitus is a risk factor for cancer, ESRD patients with diabetes showed low risk for new cancer in the previous study as well as our study. The reason may be that patients with diabetes have a relatively short life span and less time for cancer development. However, the incidence of site-specific cancer was different than in our current study, and the mean duration to new cancer was shorter in the Taiwan study than in ours. Although this discrepancy cannot be explained clearly, the authors described the effects of a Chinese herbal product on hepatitis $\mathrm{B}$ and $\mathrm{C}$ endemic areas.

Patients receiving dialysis are at a potentially increased risk of cancer compared with the general population [7,8,10-13]. Potential mechanisms of malignant transformation included impaired immune system and DNA repair, chronic infection and inflammation, medication, accumulation of carcinogen, and dialysis-related 
factors [12]. However, the reported results are not consistent $[4,14]$. Lin et al. [10] recently published a nationwide cohort study to investigate the incidence of cancer in dialysis patients matched with non-dialysis patients according to age, sex, and index year. The dialysis group had a significantly higher 7-year cancer incidence rate than the control group (6.4\% vs. $1.7 \%$ ).

It was not possible to directly compare the cancer incidence in our study with the general population incidence because we did not select a control group. However, the cumulative cancer incidence of $7.82 \%$ was relatively higher than in previous Korean studies $[3,4]$. Cancer screening and treatment of dialysis patients are important emerging medical issues because of the increasing number of older patients with ESRD. Although national cancer screening programs are well established in Korea, cancer screening in ESRD patients should be considered differently. The incidence of kidney and urinary tract cancers was higher in ESRD patients than in the general population. We suggest adding a tool for kidney and urinary tract cancers in the screening program.

This study has several limitations. First, NHID is not established for research purposes but for medical service claims and reimbursement, and the data could not confirm pathological reports for a diagnosis of cancer. If cancer diagnosis is defined as only diagnosis of $\mathrm{C}$ code, cancer incidence is increased than that of real world. However, we defined cancer diagnosis as satisfying both a new claim for major diagnosis of $\mathrm{C}$ code and V193 (special certification for cancer). Thus, we do not believe that the incidence is overestimated, as a strict cancer definition was used. Second, our results cannot be directly compared with the general population because we did not have a control group. However, this is the largest epidemiological study of cancer in ESRD patients on chronic hemodialysis using a population-based database including almost the entire population of Korea.

In conclusion, these results suggest that the cancer incidence of chronic hemodialysis patients is relatively high. Careful monitoring and a specific cancer screening program are needed. Based on these results, we plan to perform a study to investigate treatment strategies for cancer in ESRD patients with chronic maintenance hemodialysis using the NHID in Korea.

\section{KEY MESSAGE}

1. The cancer incidence of chronic hemodialysis patients $(7.82 \%)$ was relatively high.

2. The mean duration from the start of hemodialysis to new cancer identification was $64.40 \pm$ 41.81 months.

3. Significant risk factors for the development of new cancer were old age, male sex, and liver disease.

4. Careful monitoring and a specific cancer screening program are needed for hemodialysis patients.

\section{Conflict of interest}

No potential conflict of interest relevant to this article was reported.

\section{Acknowledgments}

This study used National Health Insurance Service (NHIS) data (NHIS-2018-1-024) made by NHIS. The authors declare no conflict of interest with NHIS. The authors would like to thank the NHIS for its cooperation. We thank in the Biostatistical Consulting and Research Laboratory at Hanyang University for statistical help with this study.

\section{REFERENCES}

1. Eggers PW. Has the incidence of end-stage renal disease in the USA and other countries stabilized? Curr Opin Nephrol Hypertens 2011;20:241-245.

2. Jin DC, Yun SR, Lee SW, et al. Lessons from 30 years' data of Korean end-stage renal disease registry, 1985-2015. Kidney Res Clin Pract 2015;34:132-139.

3. Lee JE, Han SH, Cho BC, et al. Cancer in patients on chronic dialysis in Korea. J Korean Med Sci 2009;24:S95-S101.

4. Yoo KD, Lee JP, Lee SM, et al. Cancer in Korean patients with end-stage renal disease: a 7-year follow-up. PLoS One 2017;12:e0178649.

5. Seong SC, Kim YY, Khang YH, et al. Data resource profile: the National Health Information Database of the National Health Insurance Service in South Korea. Int J Epidemiol 2017;46:799-800. 
6. Sundararajan V, Henderson T, Perry C, Muggivan A, Quan H, Ghali WA. New ICD-10 version of the Charlson comorbidity index predicted in-hospital mortality. J Clin Epidemiol 2004;57:1288-1294.

7. Maisonneuve P, Agodoa L, Gellert R, et al. Cancer in patients on dialysis for end-stage renal disease: an international collaborative study. Lancet 1999;354:93-99.

8. Butler AM, Olshan AF, Kshirsagar AV, et al. Cancer incidence among US medicare ESRD patients receiving hemodialysis, 1996-2009. Am J Kidney Dis 2015;65:763-772.

9. Chien CC, Han MM, Chiu YH, et al. Epidemiology of cancer in end-stage renal disease dialysis patients: a national cohort study in Taiwan. J Cancer 2017;8:9-18.

10. Lin MY, Kuo MC, Hung CC, et al. Association of dialysis with the risks of cancers. PLoS One 2015;10:e0122856.
11. Lin HF, Li YH, Wang CH, Chou CL, Kuo DJ, Fang TC. Increased risk of cancer in chronic dialysis patients: a population-based cohort study in Taiwan. Nephrol Dial Transplant 2012;27:1585-1590.

12. Vamvakas S, Bahner U, Heidland A. Cancer in end-stage renal disease: potential factors involved editorial. Am J Nephrol 1998;18:89-95.

13. Shebl FM, Warren JL, Eggers PW, Engels EA. Cancer risk among elderly persons with end-stage renal disease: a population-based case-control study. BMC Nephrol 2012;13:65.

14. Birkeland SA, Lokkegaard H, Storm HH. Cancer risk in patients on dialysis and after renal transplantation. Lancet 2000;355:1886-1887. 